\title{
Prognostic significance of fascin expression in advanced colorectal cancer: an immunohistochemical study of colorectal adenomas and adenocarcinomas
}

\author{
Yosuke Hashimoto ${ }^{1}$, Marek Skacel ${ }^{2}$, Ian C Lavery ${ }^{3}$, Abir L Mukherjee ${ }^{4}$, Graham Casey ${ }^{3,45}$ and \\ Josephine C Adams ${ }^{1,2,5^{*}}$
}

Correction [1]: It was recently brought to our attention that, although the statistical analyses in Table four had passed the original peer-review of this paper, the risk ratio and value of the lower 95\% confidence interval for some of the variables in Table four were not consistent with the $p$ values. We have repeated the multi-variant statistical analysis of the original data with StatView software. This analysis produced different values for the risk

Table 1 Corrected table four

\begin{tabular}{llc}
\hline \multicolumn{3}{l}{ Table 4a. Cox's proportional hazard analysis in stage I-IV patients } \\
\hline Variables & $\begin{array}{l}\text { Risk ratio (95\% confidence } \\
\text { Interval) }\end{array}$ & $\boldsymbol{p}$ \\
\hline Age ( $\geq 65$ yrs) & $1.618(0 / 944-2.774)$ & 0.080 \\
Gender (male) & $1.626(0.965-2.740)$ & 0.068 \\
Lymph node metastasis & $0.253(0.146-0.439)$ & $<$ \\
(N1, 2) & & $0.001^{*}$ \\
Distant metastasis (M1) & $0.581(0.317-1.066)$ & 0.797 \\
Tumour location (distal) & $0.963(0.550-1.686)$ & 0.894 \\
Fascin (high) & $0.548(0.272-1.104)$ & 0.925 \\
\hline Table 4b. Cox's proportional hazard analysis in stage III/IV patients \\
\hline Variable & Risk ratio (95\% confidence & $p$ \\
& Interval) & 0.460 \\
\hline Age (> 65 yrs) & $1.259(0.683-2.318)$ & 0.114 \\
Gender (male) & $1.659(0.886-3.108)$ & 0.934 \\
Lymph node metastasis & $1.032(0.358-2.972)$ & 0.566 \\
(N1, 2) & & 0.240 \\
Distant metastasis (M1) & $0.803(0.381-1.697)$ & $0.039^{*}$ \\
Tumour location (distal) & $0.683(0.362-1.289)$ & \\
Fascin (high) & $0.435(0.197-0.957)$ & \\
\hline & & \\
\hline
\end{tabular}

ratios and 95\% confidence intervals for variables other than age. The corrected Table four is presented below (table 1). The $p$ values are unchanged, thus the original conclusions of the article are not altered.

\section{Author details \\ 'Department of Cell Biology, Lerner Research Institute, The Cleveland Clinic, 9500 Euclid Avenue, Cleveland, OH 44195, USA. ${ }^{2}$ Department of Anatomic Pathology, The Cleveland Clinic, 9500 Euclid Avenue, Cleveland, OH 44195, USA. ${ }^{3}$ Department of Colorectal Surgery, The Cleveland Clinic, 9500 Euclid Avenue, Cleveland, OH 44195, USA. ${ }^{4}$ Department of Cancer Biology, Lerner Research Institute, The Cleveland Clinic, 9500 Euclid Avenue, Cleveland, $\mathrm{OH}$ 44195, USA. ${ }^{5}$ Department of Molecular Medicine, Cleveland Clinic Lerner College of Medicine at Case Western Reserve University, The Cleveland Clinic, 9500 Euclid Avenue, Cleveland, OH 44195, USA.}

Received: 15 November 2011 Accepted: 21 November 2011

Published: 21 November 2011

\section{Reference}

1. Hashimoto Y, Skacel M, Lavery IC, Mukherjee AL, Casey G, Adams JC: Prognostic significance of fascin expression in advanced colorectal cancer: an immunohistochemical study of colorectal adenomas and adenocarcinomas. BMC Cancer 2006, 6:241.

\section{Pre-publication history}

The pre-publication history for this paper can be accessed here: http://www.biomedcentral.com/1471-2407/11/488/prepub

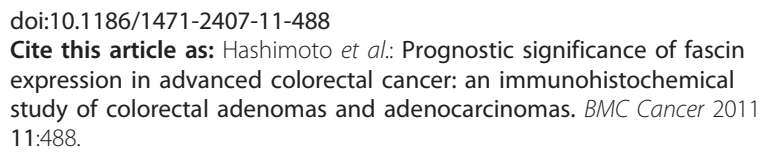

Cite this article as: Hashimoto et al:: Prognostic significance of fascin expression in advanced colorectal cancer: an immunohistochemical study of colorectal adenomas and adenocarcinomas. BMC Cancer 2011 11:488.

\footnotetext{
* Correspondence: jo.adams@bristol.ac.uk

'Department of Cell Biology, Lerner Research Institute, The Cleveland Clinic, 9500 Euclid Avenue, Cleveland, OH 44195, USA

Full list of author information is available at the end of the article
} 